\title{
Rückenschmerzen
}

\section{Der Chronifizierung frühzeitig entgegenwirken}

_ Die Chronifizierung von Rückenschmerzen ist ein komplexer Vorgang, bei dem die periphere und die zentrale Sensibilisierung sowie die Abnahme der körpereigenen Schmerzhemmung eine wichtige Rolle spielen, führte Prof. Ralf Baron, Kiel, aus. Die periphere Sensibilisierung führe $\mathrm{zu}$ einem ständigen Feuern der Schmerzfaser, was u. a. die zentrale Sensibilisierung unterhalte. Diese wiederum führe zu einer Hyperaktivität des Rückenmarks. Durch diese Prozesse nehme zusätzlich die Wirkung der körpereigenen noradrenergen Schmerzhemmung kontinuierlich ab, betonte Baron.

Unter den zur Verfügung stehenden Schmerzmitteln wirke Tapentadol $\left(\right.$ Palexia $\left.{ }^{\oplus}\right)$ als einziger Wirkstoff auf alle drei Faktoren der Schmerzchronifizierung ein, sagte der Neurologe. Der Wirkstoff reduziere die Weiterleitung der Schmerzsignale und stärke aufgrund seines dualen Wirkmechanismus gleichzeitig die körpereigene absteigende Schmerzhemmung. „Für mich bedeutet das, dass man mit dem frühen Einsatz eine Chronifizierung eventuell verhindern kann“, sagte Baron.

Neben der medikamentösen Therapie können Schmerztherapeuten gemeinsam mit den Patienten den Chronifizierungsprozess positiv beeinflussen. Wichtig sei eine gute Arzt-Patienten-Beziehung mit einer empathischen Ansprache, betonte PD Dr. Kai-Uwe Kern, Schmerzpraxis Wiesbaden. Eher kontraproduktiv seien Sätze wie „Das verstehen Sie nicht, ich mache das schon.“ Stattdessen empfiehlt Kern die direkte Einbindung des Patienten: „Lassen Sie uns gemeinsam schauen, welche Bausteine Ihr Schmerz haben könnte und welche wir als erste gemeinsam angehen wollen."

Wichtige Faktoren der Schmerzchronifizierung aus psychologischer Sicht sind eine ungünstige Einstellung zum sowie ein vermeidender oder überaktiver Umgang mit Schmerz, sagte Dipl.Psych. Patricia Albert vom Schmerzzentrum des Universitätsklinikums Erlangen. Ein adaptiver Umgang mit Schmerzen sei zum einen gekennzeichnet durch

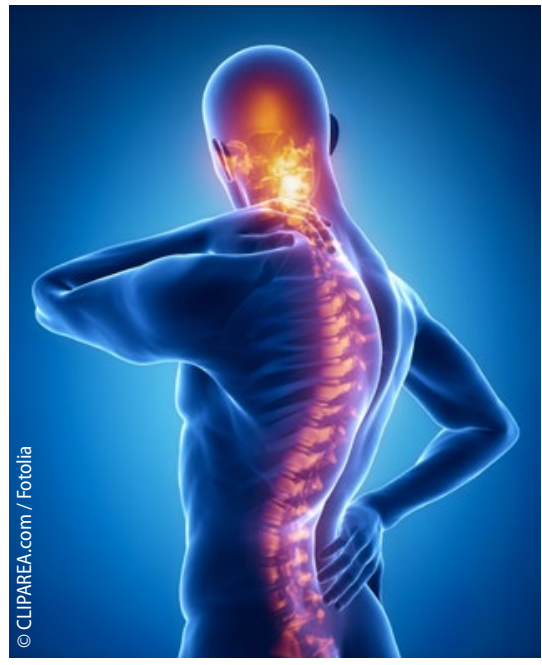

Rückenschmerzen können auch im Kopf entstehen.

die Gewissheit, trotz Schmerzen Einfluss auf das eigene Leben zu haben, und zum anderen die Bereitschaft, negative innere Erfahrungen zuzulassen. Erfah rungsvermeidung stelle dabei das größte Risiko für dysfunktionales Verhalten dar.

Dr. Gunter Freese

- Lunchsymposium „Einfluss der Schmerztherapie auf den Chronifizierungsprozess", Deutscher Schmerzkongresses 2016; Mannheim, Oktober 2016 (Veranstalter: Grünenthal)

\section{Rhinosinusitis}

\section{Phytopharmakon beschleunigt Heilung}

_ Eine aktuelle Zusammenfassung klinischer Daten zu BNO 1016, einer FünfPflanzen-Kombination auf Extraktbasis, bestätigt deren Wirksamkeit gegen typische Symptome der Rhinosinusitis [Ammendola A, Bachert C. Pharmakon. 2016;4:74-82]. Unter der Therapie mit dem Phytopharmakon (Sinupret ${ }^{\circledR}$ extract) kommt es u.a. zu einem rascheren Abklingen des Druckkopfschmerzes: In einer Studie waren die Patienten nach einer Woche Therapie im Vergleich zur Placebogruppe zwei Tage schneller wieder fit. Sowohl Ärzte als auch Patienten beurteilten dabei die Verträglichkeit mit gut bzw. sehr gut.
Viele Patienten spüren die starke Wirkung des pflanzlichen Arzneimittels recht schnell. Dies zeigt $z$. B. eine Umfrage unter HNO-Ärzten, die nach Einnahme von BNO 1016 über eine spürbare Wirkung bereits innerhalb von 30 Minuten berichteten.

Wichtig zu wissen, wenn Ärzte zu einem Phytopharmakon raten: Laut einer Umfrage des Bundesverbands der pharmazeutischen Industrie (BPI) erwarten zwei Drittel der Patienten, dass ihnen das beste Medikament verordnet wird - und nicht das günstigste Produkt.

Red.

- Nach Informationen von Bionorica

\section{Kurz notiert}

Laktoseintoleranz $\rightarrow$ Neben diätetischen Maßnahmen kann bei Laktoseintoleranz eine Enzymsubstitution sinnvoll sein. Hierfür steht das Nahrungsergänzungsmittel Laluk ${ }^{\circledast} 4500$ mit 4.500 FCC-Einheiten Laktase pro Kapsel zur Verfügung. Sie können ca. 10-20 g Laktose spalten. Laluk ${ }^{\circledast} 4500$ lässt sich entweder als Kapsel einnehmen, oder man kann deren Inhalt der Nahrung beimengen. So kann vielen Patienten, deren uncharakteristische Bauchschmerzen auf einer Laktose-Intoleranz beruhen, geholfen werden. Schließlich sind Milch und Milchprodukte wichtige Kalziumlieferanten, die der Körper für ein stabiles Knochenwachstum braucht. Weitere Informationen finden Sie unter www.laluk.de.

Red.

- Nach Informationen von Strathmann 